\title{
Generalization slope as a function of the density of variable interval reinforcement
}

\author{
GREGORY A. DAVITT, JAMES F. DICKSON, JR., KIMBAL L. WHEATLEY, and DAVID R. THOMAS \\ University of Colorado, Boulder, Colorado 80302
}

\begin{abstract}
Five groups of 10 pigeons each were trained to peck a vertical line under VI 14-sec, 21-sec, 35-sec, $56-\mathrm{sec}$, and 4-min reinforcement schedules and then were tested for angularity generalization in extinction. Both absolute and relative generalization gradients were sharpest with the intermediate schedule densities and flattest following training with the richest and the leanest schedules. Only the differences based upon relative gradients achieved statistical significance however.
\end{abstract}

One of the variables known to influence the slope of generalization gradients is the schedule of reinforcement used during training. Haber and Kalish (1963) and Hearst, Koresko, and Poppen (1964) investigated generalization as a function of the reinforcement density of VI schedules. In both studies it was concluded that the generalization slope varies positively with reinforcement density. In explanation of this finding, Hearst et al. speculated that leaner VI schedules encourage the development of superstitious chaining of responses, thereby enhancing the role of proprioceptive feedback. It may be argued, however, that the greater the reinforcement density of the VI schedule, the greater the opportunity for food delivery and resultant consummatory activities with their feedback to gain control over key-pecking. Furthermore, higher response rates generated by richer schedules might provide still an additional source of proprioceptive stimuli. It might be expected, therefore, that the relationship between generalization slope and VI density would be nonmonotonic. Indeed, Hearst, Koresko, and Poppen (1964) obtained data that suggest such a function, with a VI $30-s e c$ schedule producing a nonsignificantly flatter gradient than a VI 60-sec schedule. In both the Haber and Kalish (1963) and Hearst et al. (1964) experiments, only one VI schedule richer than $60 \mathrm{sec}$ was employed, thus the nature of the relationship between generalization slope and VI reinforcement density (in this region) needs to be more precisely determined. THis was the purpose of the present experiment.

\section{METHOD}

\section{Subjects}

Fifty adult domestic pigeons, maintained at approximately $75 \%$ of their free-feeding weights, wrere used in this experiment.

This research was supported in part by NIH Research Grant HD-03486 and Training Grant MH-10427. At the time of the investigation, James F. Dickson, Jr. was on sabbatical leave from St. Olaf College. Kimbal L. Wheatley is now at Weber State College. Requests for reprints should be sent to David $R$. Thomas, Department of Psychology, University of Colorado, Boulder, Colorado 80302.

\section{Apparatus}

Four standard single key operant conditioning chambers were employed with all experimental conditions controlled by electromechanical equipment located in an adjacent room.

Stimuli used were white lines on a black background projected onto the key by I.E.E. in-line display cells. The lines were $.32 \mathrm{~cm}$ wide $\times 2.24 \mathrm{~cm}$ long and bisected the key at angles of $90 \mathrm{deg}$ (vertical), 75, 60, 45, 30, 15, and $0 \mathrm{deg}$. White noise was present in the chambers at all times.

\section{Procedure}

The subjects were randomly assigned to one of five groups which differed only in the reinforcement schedule that was operative during training. The schedules investigated were variable interval (VI) $14 \mathrm{sec}, 21 \mathrm{sec}, 35 \mathrm{sec}, 56 \mathrm{sec}$, and $4 \mathrm{~min}$ constructed according to the progression suggested by Catania and Reynolds (1968, p. 381). On Day 1 of training all subjects were first shaped to keypeck and then given 50 reinforcements on a continuous reinforcement (CRF) schedule. On the second and third days of training, all subjects were again given $50 \mathrm{CRF}$. During the fourth session, subjects in the first four groups (those with VI schedules of 14 through $56 \mathrm{sec}$ ) were switched to their appropriate schedules for $20 \mathrm{~min}$. On their fourth session, subjects in the VI 4-min group were given intermediate values of VI density, and were placed on a VI 4-min schedule by the fifth session. This was done to lessen the abrupt change from CRF to a 4-min schedule. Beginning with Day 5, daily sessions for all subjects were $20 \mathrm{~min}$ in length.

Throughout training, the translucent key was constantly illuminated with a vertical white line on a black surround, and the reinforcing event consisted of $2 \mathrm{sec}$ of access to a tray of mixed grain. The only sources of illumination in the chambers were the food hopper light when reinforcement was delivered and the key stimulus.

After 16 sessions on their respective VI schedules, subjects were tested for angularity generalization in extinction following a 10-min warm-up period under the normal training condition. Test stimulus periods were $60 \mathrm{sec}$ long and were arranged in six randomly permuted blocks of the seven stimuli, with no intervening blackouts.

\section{RESULTS}

One consequence of variations in VI reinforcement density is resultant differences in response rate. As expected, response rate was positively related to reinforcement density in this experiment. The mean response rates averaged over the last 3 days of training were $105.5,60.4,45.7,50.4$, and 28.0 responses $/ \mathrm{min}$ 
(corrected for reinforcement time) for the VI 14-sec, 21 -sec, 35-sec, 56-sec and 4-min schedules, respectively. An analysis of variance indicated the group differences to be, significant $(\mathrm{F}=10.22$, df $-4 / 45, \mathrm{p}<.01)$. Thus, the richest schedule resulted in high rates of response, providing a postulated source of interoceptive cues that could interfere with exteroceptive stimulus control.

It has become common practice in operant free-response studies to report generalization gradients in relative terms, e.g. percent of total responses emitted to the various test stimuli (cf. Thomas, Freeman, Svinicki, Burr, \& Lyons, 1970). This transformation eliminates the typically large individual differences in response rates, thereby permitting each subject to contribute equally to the average gradient of its group. Relative gradients, therefore, constitute the primary data of the present study: however, absolute gradients are also presented.

The mean relative generalization gradients are plotted for each of the five groups in Figure 1. The training stimulus (the 90-deg line orientation) is indicated by the abbreviation CS. The sharpest gradient, with $31.1 \%$ of total responses to the CS, was that of the VI $35-\mathrm{sec}$ group. The flattest gradients came from the two extreme conditions, the 14-sec and 4-min groups, which yielded gradients with $19.3 \%$ and $20.4 \%$ of total responses to the CS, respectively. The intermediate values of sciredule density resulted in gradients intermediate to the above extremes, with $22.8 \%$ and $22.3 \%$ of total responses made to the CS for the 21-sec and 56-sec groups, respectively.

An analysis of variance was carried out with the data presented in Figure 1. The result was a significant effect of stimulus $(\mathrm{F}=41.32, \mathrm{df}=6 / 720, \mathrm{p}<.01)$ and $\mathrm{a}$ significant Stimulus by Group interaction $(\mathrm{F} \cdot 1.56 \mathrm{df}=$ $24 / 270, p<.05$ ), the latter value indicating that the relative gradients differed reliably in slope. Because a quadratic relationship was predicted between VI density and generalization slope, a quadratic trend analysis of the data was performed. For this purpose, a single score was needed to represent the slope of each gradient. The score selected was the percent of total responses given to the CS value: the higher this score, the shaper the gradient. Assuming that reinforcement density is psychophysically logarithmic, the schedule values were converted into natural logarithms for use in this trend analysis. The schedule values used only approximated equal log spacing, so a trend analysis for unequal spacing (Gaito, 1964) was employed. This analysis resulted in a significant quadratic effect $(\mathrm{F}=6.33, \mathrm{df}=1 / 45$, $\mathrm{p}<.025)$, indicating that the quadratic function relating generalization slope to VI schedule density is a reliable one.

It is well known that generalization gradients tend to sharpen during the course of extinction during prolonged generalization testing (cf. Jenkins \& Harrison, 1960; Friedman \& Guttman, 1965). It is therefore important to determine whether the group differences in

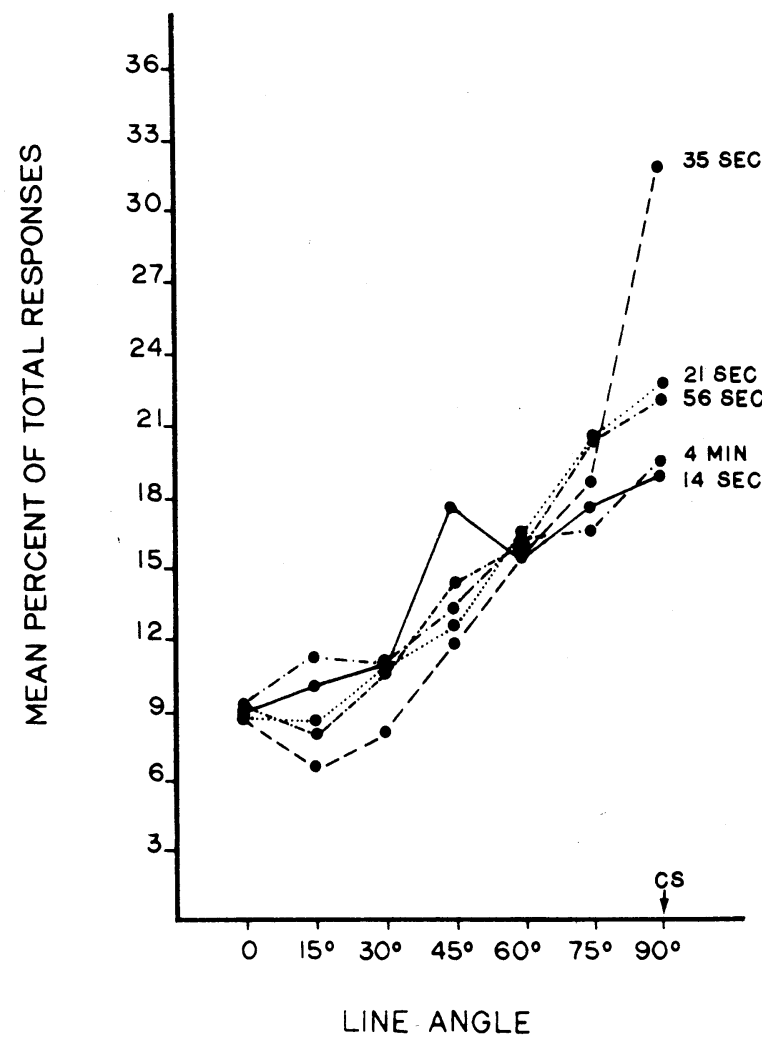

Figure 1. Mean relative line angle generalization gradients.

generalization slope reported here are a consequence of possible differences in resistance to extinction produced by the different training schedules. For this purpose, the data were reanalyzed in terms of the first three series of generalization test stimuli rather than all six. The results were quite comparable to those obtained with the entire test. Again the VI 35-sec group produced the sharpest gradient, with $30.6 \%$ of total responses to the CS. The VI 21 -sec and VI 56-sec conditions followed, with means of 20.6 and 21.6, respectively, and the VI 14-sec and VI 4-min gradients were the flattest, at $18.6 \%$ and $19.5 \%$. Analysis of variance indicated both a significant effect of stimuli $(\mathrm{F}=34.03, \mathrm{df}=6 / 270, \mathrm{p}<.01)$ and a signfiicant Stimulus by Group interaction $(\mathrm{F}=1.62, \mathrm{df}=24 / 270$, $p<.05)$; denoting that halfway through the generalization test the groups differed in gradient slope. Again a trend analysis performed on the percent of total responses to the $\mathrm{CS}$ indicated a significant quadratic component $(F=7.89$, df $-1 / 45, p<.01)$. Thus, the obtained differences in relative generalization slope were not an artifact of group differences in resistance to extinction.

Figure 2 presents the mean absolute generalization gradients of the five groups of subjects. The absolute gradients are variable in form and differ in height (i.e., response level) more clearly than they do in slope. An analysis of variance indicated that neither the effect of 
group nor the Stimulus by Group interaction approached significance ( $\mathrm{F}<1$ in both cases). The lack of statistical significance in the comparison of absolute gradient slopes is typical in studies of this sort, accounting in part for the reliance upon relative measures. However, visual inspection of the gradient of the VI 35-sec group and those of the VI 14-sec and VI 4-min groups indicates differences consistent with the relative measures, with the VI $35-\mathrm{sec}$ gradient sharper than (and intersecting) both of the other two.

\section{DISCUSSION}

Subsequent to the performance of the present experiment a replication and extension was completed. Three groups of 10 pigeons each were trained with VI 14-sec, VI 35-sec, and VI 4-min schedules, respectively, to peck a key illuminated by a 555-nm light. A subsequent wavelength generalization test administered in extinction confirmed the pattern of results reported earlier for the angularity dimension. Again the data revealed a signficant quadratic effect of VI schedule density on relative generalization slope. Again, although the absolute generalization gradient of the VI 35-sec group was sharper than that of the other two groups, these absolute differences did not achieve statistical significance.

Thus the results of these two experiments are consistent with the hypothesis that generalization slope varies nonmonotonically with density of VI schedule. Since these results are inconsistent with the conclusions of the Hearst et al. (1964) and Haber and Kalish (1965) studies, a comparison of the data of the relevant studies is warranted. All the experiments are in agreement that gradients flatten with a decrease in reinforcement density beyond VI 1-min. Like the present study, that of Hearst et al. suggests a nonmonotonic function. Those investigators found a (nonsignificantly) flatter gradient for VI 30-sec than for VI 1 -min. This is inconsistent, however, with Haber and Kalish's finding of a sharper gradient for Vi 15-sec than for VI 1-min. Quite possibly Haber and Kalish would have found a maximally sharp gradient at some value between 15 and $60 \mathrm{sec}$, as was the case in the present experiments. A more direct comparision of the various experiments is difficult because of many procedural differences, including different VI values, different stimulus dimensions and test values, and the use of blackouts in the earlier studies. Assuming that a nonmonotonic (i.e., quadratic) function is involved here, its inflection point might vary with any one or combination of these factors.

Our findings are consistent with the hypothesis of a trading relationship between key stimuli and other stimuli if the assumption is granted that both rich and lean VI schedules provide for greater competition with key stimuli than do schedules with intermediate reinforcement density. We have made this assumption and have further proposed that the specific sources of competing stimuli are different in the two kinds of extreme schedule densities. In rich VI schedules, high rates of responses are generated providing enhanced proprioceptive feedback from repetitive responding. In such schedules, the reinforcing event itself and the feedback associated with behavior generated by it provide additional salient stimuli. With lean schedules, on the other hand, elaborate chains of collateral behavior may develop (cf. Hearst et al., 1964).

We realize that neither collateral behavior nor proprioceptive

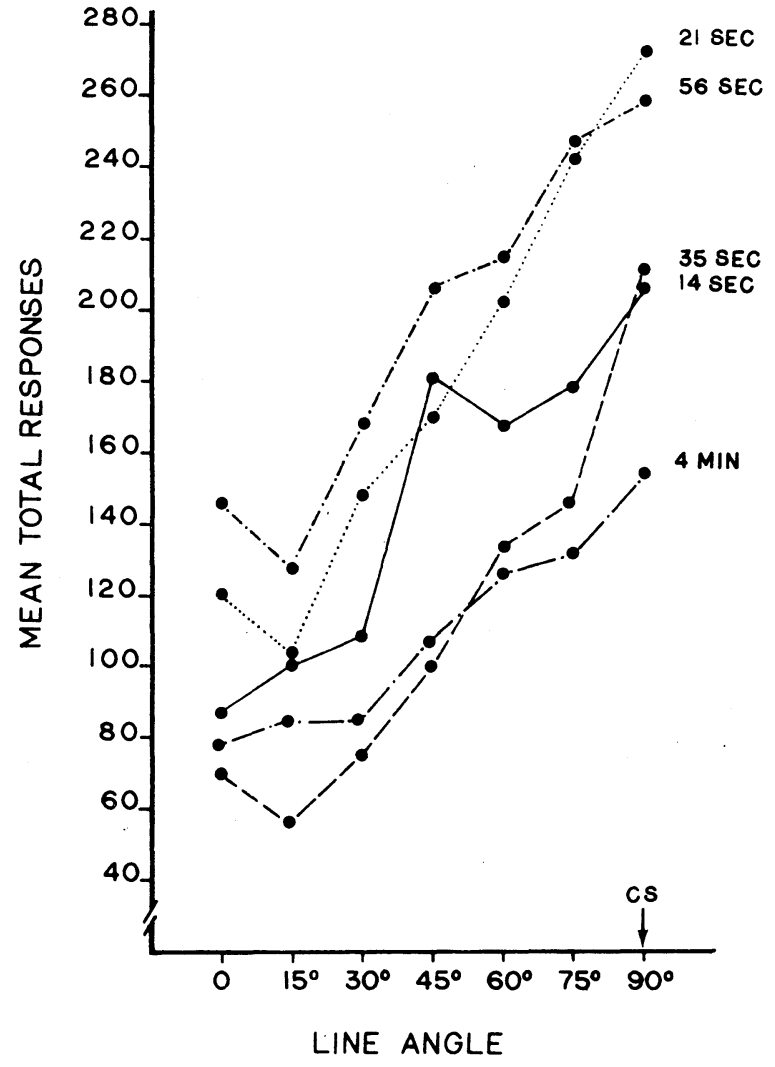

Figure 2. Mean absolute line angle generalization gradients.

feedback were directly observed or measured in this study, and in the absence of such measures our interpretation is admittedly inferential. Nevertheless that interpretation led us to predict the obtained pattern of results and we are aware of no other position that would have done so.

\section{REFERENCES}

Catania, A. C., \& Reynolds, G. S. A quantitative analysis of the responding maintained by interval schedules of reinforcement. Journal of the Experimental Analysis of Behavior, 1968, 11, 327-383.

Friedman, H., \& Guttman, N. Further analysis of the various effects of discrimination training on stimulus generalization gradients. In D. I. Mostofsky (Ed.) Stimulus generalization. Palo Alto: Stanford University Press, 1965, Pp. 255-267.

Gaito, J. Unequal intervals and unequal $n$ in trend analysis. Psychological Bulletin, 1964, 63, 125-127.

Haber, A., \& Kalish, H. I. Prediction of discrimination from generalization from variations in schedule of reinforcement. Science, 1963, 142, 412-413.

Hearst, E., Koresko, M. B., \& Poppen, R. Stimulus generalization and the response-reinforcement contingency. Journal of the Experimental Analysis of Behavior, 1964, 7, 369-380.

Jenkins, H. M., \& Harrison, R. H. Effect of discrimination training on auditory generalization. Journal of Experimental Psychology, 1960, 59, 246-253.

Thomas, D. R., Freeman, F., Svinicki, J. G., Burr, D. E. S., \& Lyons, J. Effects of extradimensional training on stimulus generalization. Journal of Experimental Psychology, 1970, 83, ( Pt. 2).

(Received for publication October 29, 1974.) 